\title{
ORIGINAL Clinical and Angiographic Characteristics of Multiple Dural Arteriovenous Shunts \\ RESEARCH
}

\author{
S.Y. Ha
}

Y.S. Kwon

B.M. Kim

D.I. Kim

D.J. Kim

\begin{abstract}
BACKGROUND AND PURPOSE: The pathogenesis and characteristics of multiple DAVSs are not wellknown. The purpose of this study was to evaluate the angiographic and clinical characteristics of patients with multiple DAVSs with an emphasis on the pathomechanism.
\end{abstract}

MATERIALS AND METHODS: One hundred seventy-nine patients with DAVS were reviewed. Patients with $\geq 2$ fistulas at anatomically separate sites were included. Multiple DAVSs were categorized into synchronous (simultaneous multiplicity) or metachronous (temporal sequential development of multiplicity) types. The angiographic and clinical characteristics of these lesions were analyzed.

RESULTS: Fourteen patients were diagnosed with multiple DAVSs $(7.8 \%$; synchronous, $n=7$; metachronous, $n=7)$. Thirteen of the 14 patients showed CVR (93\%, Borden type II/III). Multiple DAVSs were frequently associated with dural sinus thrombosis $(71.4 \%, n=10)$. Synchronous DAVSs developed in association with an occluded sinus $(n=5)$. De novo metachronous lesions developed in association with thrombosis of a previously patent dural sinus $(n=3)$ or reopening of an occluded sinus $(n=2)$. Multiplicity was associated with aggressive initial symptoms in $64.3 \%(n=9)$. The newly developed lesions in the metachronous types were accompanied by hemorrhage $(n=1)$, neurologic deficit ( $n=1)$, worsening of the initial benign symptoms $(n=2)$, and incidental detection $(n=3)$. The mean time interval between the initial diagnosis and de novo lesion detection was $31.3 \pm 29.8$ months (range, 12-92 months).

CONCLUSIONS: Multiplicity of DAVSs is associated with poor angiographic and clinical prognosis, requiring an aggressive treatment and management strategy. Sinus thrombosis has a prominent role in the pathomechanism of DAVSs.

ABBREVIATIONS: CS = cavernous sinus; CVR = cortical venous reflux; DAVS $=$ dural arteriovenous shunt; $M S=$ marginal sinus; $S S=$ sigmoid sinus; $S S S=$ superior sagittal sinus; $T S=$ transverse sinus
D AVS is a disease in which abnormal arteriovenous communications develop within the dura. These are dynamic lesions and can show spontaneous healing or pattern changes on follow-up DSA. ${ }^{1,2}$ DAVSs compose approximately $10 \%$ of all intracranial vascular malformations. ${ }^{3}$ They are generally considered to be acquired diseases in the adult as opposed to other intracranial vascular malformations, but their pathomechanism in terms of lesion development and temporal changes has not been clearly established. A wide variety of factors, such as infections, surgery, postpartum status, and various coagulopathies, may provide a conducive environment for disease development. ${ }^{1,4,5}$ Angiogenic growth factors and sinus thrombosis associated with venous hypertension have also been implicated in the pathogenesis of DAVS.,6

Only a few articles with a small number of cases have been reported concerning multiple DAVSs. Multiplicity of DAVSs can be seen simultaneously, or a de novo lesion may develop in a temporal sequence. ${ }^{7-20}$ The angiographic and clinical characteristics of these lesions need further elucidation, and an

Received December 1, 2011; accepted after revision January 14, 2012

From the Department of Radiology, Yonsei University College of Medicine, Seoul, Korea.

This work was supported by a grant from the Korea Healthcare Technology R\&D Project, Ministry for Health and Welfare Affairs, Republic of Korea (A085136).

Please address correspondence to Dong Joon Kim, MD, PhD, Department of Radiology, Severance Hospital, Yonsei University College of Medicine, 50 Yonsei-ro, Seodaemun-gu, Seoul 120-752, South Korea; e-mail: djkimmd@yuhs.ac

Indicates open access to non-subscribers at www.ajnr.org

http://dx.doi.org/10.3174/ajnr.A3054 investigation of the multiplicity of DAVSs may provide new understanding in the pathogenesis and the pathomechanism of this dynamic disease.

The purpose of this study was to analyze the angiographic and clinical characteristics of multiple DAVSs with special interest in the pathomechanism.

\section{Materials and Methods}

\section{Patients}

The patients were selected from a single institution data base consisting of 194 cases diagnosed as or treated for intracranial DAVSs between January 2000 and February 2011. The data have been collected prospectively since 1980 in a dedicated neurointerventional data base. The diagnosis of DAVS was based on DSA findings. Patients without DSA $(n=15)$ were excluded from this study. The imaging findings of DSA, CT, MR imaging, and medical records of 179 patients were reviewed. Institutional review board approval was obtained for this retrospective analysis with a waiver of informed consent.

\section{Imaging and Clinical Analysis}

The DSA images were investigated for multiplicity, location, CVR, treatment method/result, and sinus patency. CVR was classified according to the Borden classification: type I, drainage directly into the dural venous sinuses or meningeal veins; type II, drainage into the dural sinuses or meningeal veins but also with retrograde drainage into the subarachnoid veins; and type III, drainage into the subarachnoid veins without dural sinus or meningeal venous drainage. ${ }^{21}$ "Multiplicity" was defined as the occurrence of $\geq 2$ fistulas at anatom- 


\begin{tabular}{|c|c|c|c|c|c|c|}
\hline \multirow{2}{*}{$\begin{array}{l}\text { Case } \\
\text { No. }\end{array}$} & \multirow{2}{*}{$\begin{array}{c}\text { Sex/Age } \\
\text { (yr) }\end{array}$} & \multirow[b]{2}{*}{ Initial Presentation } & \multirow[b]{2}{*}{ Location } & \multicolumn{2}{|l|}{ Borden } & \multirow[b]{2}{*}{ Treatment/Result } \\
\hline & & & & Type & Sinus Patency & \\
\hline \multirow[t]{2}{*}{1} & $F / 53$ & Headache, tinnitus, chemosis & CS, left & $\|$ & Occluded IPS & $\overline{T A E}, \mathrm{TVE} / \mathrm{Po}_{0}$ \\
\hline & & & MS, right & I & Occluded MS & TVE/Co \\
\hline \multirow[t]{2}{*}{2} & $M / 54$ & Gait disturbance & SS, left & $\|$ & Occluded SS & TVE/Co \\
\hline & & & SSS & III & Patent SSS & TAE/Co \\
\hline \multirow[t]{2}{*}{3} & $\mathrm{~F} / 59$ & Aphasia, hemianopsia & SS, left & $\|$ & Near-occluded TS, left & TAE/Co \\
\hline & & & TS, right & I & Patent TS, right & TAE/Po \\
\hline \multirow[t]{2}{*}{4} & $\mathrm{M} / 42$ & Seizure & SS, right & $\|$ & Isolated SS & TVE/Co \\
\hline & & & Frontal convexity, left & III & & $\mathrm{TAE} / \mathrm{Co}_{0}$ \\
\hline \multirow[t]{2}{*}{5} & $\mathrm{M} / 58$ & $\mathrm{Hb}$ & TS, left & $\|$ & Patent TS & TVE, Onyx/Co \\
\hline & & & Jugular bulb, left & I & Isolated sinus & None \\
\hline \multirow[t]{2}{*}{6} & $M / 59$ & Exophthalmoses & TS, right & $\|$ & Patent TS & TAE, TVE/Co \\
\hline & & & MS, right & I & Patent MS & TAE, TVE/Co \\
\hline \multirow[t]{3}{*}{7} & $M / 49$ & Right arm weakness, aphasia & SSS & $\|$ & Patent SSS & None \\
\hline & & & TS, right & । & Patent TS & None \\
\hline & & & SS, right & I & Patent SS & None \\
\hline
\end{tabular}

Note:- Co indicates complete occlusion; Hb, hemorrhage; IPS, inferior petrosal sinus; Po, partial occlusion; TAE, transarterial embolization; TVE, transvenous embolization.

\begin{tabular}{|c|c|c|c|c|c|c|c|}
\hline $\begin{array}{l}\text { Case } \\
\text { No }\end{array}$ & $\begin{array}{l}\text { Sex/Age } \\
\text { (yr) }\end{array}$ & $\begin{array}{c}\text { Initial Presentation (1)/ } \\
\text { De Novo Lesion Presentation (2) }\end{array}$ & Location & $\begin{array}{l}\text { Borden } \\
\text { Type }\end{array}$ & $\begin{array}{l}\text { Time Interval } \\
\text { (months) }\end{array}$ & Sinus Patency & Treatment/Result \\
\hline \multirow[t]{2}{*}{8} & $F / 68$ & 1) Eyeball pain, ptosis & CS, left & $\|$ & & Occluded SS & TAE/Co \\
\hline & & 2) $\mathrm{Hb}$, delayed $\mathrm{Hb}$ & SS, left & $\|$ & 17 & Recanalized SS & TAE, burr-hole/Co \\
\hline \multirow[t]{2}{*}{9} & $\mathrm{~F} / 56$ & 1) Eyeball pain, pulsatile tinnitus & CS, right & I & & Occluded IPS after TVE & TVE/Co \\
\hline & & 2) Pulsatile tinnitus & MS, left & । & 17 & Occluded IPS & TVE/Co \\
\hline \multirow[t]{2}{*}{10} & $\mathrm{M} / 17$ & 1) $\mathrm{Hb}$ & TS, right & $\|$ & & Patent torcular & TAE, TVE, GKS/Po \\
\hline & & 2) Aggravated headache & Torcular & $\|$ & 50 & Occluded torcular & Onyx/Po \\
\hline \multirow[t]{2}{*}{11} & $\mathrm{M} / 1$ & 1) Developmental delay & Falcine sinus & $\|$ & & Patent torcular & $\mathrm{TAE} / \mathrm{Co}$ \\
\hline & & 2) Routine follow-up & Torcular & $\|$ & 12 & Occluded torcular & TAE, Onyx/Co \\
\hline \multirow[t]{2}{*}{12} & $M / 28$ & 1) Seizure & SSS & $\|$ & & Occluded TS & TAE, TVE/Po \\
\hline & & 2) Right leg weakness (delayed $\mathrm{Hb})^{\mathrm{a}}$ & TS, left & I & 92 & Recanalized TS & TAE/Po \\
\hline \multirow[t]{2}{*}{13} & $M / 58$ & 1) Gait disturbance, memory dysfunction & SSS & $\|$ & & Patent TS & TAE, burr-hole/Co \\
\hline & & 2) Routine follow-up & TS, right & 1 & 20 & Patent TS & $\mathrm{TAE} / \mathrm{Co}$ \\
\hline \multirow[t]{2}{*}{14} & $\mathrm{~F} / 23$ & 1) Bruit & TS, both & $\|/\|$ & & Patent TS & TAE, TVE/Po \\
\hline & & 2) Routine follow-up & TS, left & $\|$ & 12 & Stenotic TS, left & TAE, TVE/Po \\
\hline
\end{tabular}

Note:-Co indicates complete occlusion; GKS, gamma knife surgery; Hb, hemorrhage; IPS, inferior petrosal sinus; Po, partial occlusion; TAE, transarterial embolization; TVE, transvenous embolization.

a Intraventricular hemorrhage probably associated to partially treated lesions.

ically separate sites in 1 patient. Multiple DAVSs were categorized as "synchronous" if the shunt occurred simultaneously in different dural sinuses and "metachronous" if a de novo shunt developed at a different location in a temporal sequence. Chronologic changes on DSA in patients with metachronous DAVSs were analyzed. Brain CT or MR imaging was evaluated for the presence of hemorrhage, venous hypertension, and sinus thrombosis. Clinically, the patients were generally followed on a 2- to 3-month basis for a year and 6- to 12-month intervals afterward. Imaging studies were performed during this follow-up period in reference to the treatment results and whether changes or deterioration of symptoms believed to be related to the DAVS had occurred. The clinical characteristics of the patients, such as sex, age, initial/follow-up symptoms, development of hemorrhage, and long-term outcome were analyzed.

A board-certified neuroradiologist and neurologist (D.J.K. and S.Y.H.) reviewed the angiographic and clinical findings in consensus.

\section{Results}

Fourteen of 179 patients were diagnosed as having multiple DAVSs $(7.8 \%)$ on DSA. Ages ranged from 1 to 68 years (mean age, $45 \pm 19.7$ years; male/female $=9: 5)$. The multiplicity of the 14 patients was categorized as synchronous $(n=7)$ or metachronous $(n=7)$ types. The mean duration of clinical follow-up for the metachronous lesions was 66 months (17122 months).

Thirteen of the 14 patients with multiple DAVSs showed CVR (93\%, Borden type II / III) on the initial DSA. The locations of the synchronous types were CS + MS, TS + MS, SSS + SS, SSS + SS + TS, SS + TS, TS + jugular bulb, and SS + frontal convexity, respectively (Table 1 ). The locations of the metachronous types were SSS + TS $(n=2)$, CS + SS, CS + MS, TS + torcular herophili, bilateral TS, and falcine sinus + torcular herophili, respectively (Table 2). Four of the 7 de novo DAVSs showed CVR.

Angiographically, the multiple DAVSs were frequently associated with dural sinus thrombosis $(71.4 \%, n=10)$. Synchronous DAVS developed in association with a thrombosed sinus in 5 of the 7 patients $(71.4 \%)$. In the metachronous types, 5 of the 7 de novo shunts developed in association with thrombosis of a previously patent dural sinus $(n=3$; cases 9 , 10 , and 11) or reopening of an occluded dural sinus (Fig $1 ; n=$ 2 , cases 8 and 12). In terms of temporal development, sinus thrombosis was verified on DSA before sequential de novo lesion development in 3 patients (Fig 2; cases 8, 11, and 12). 


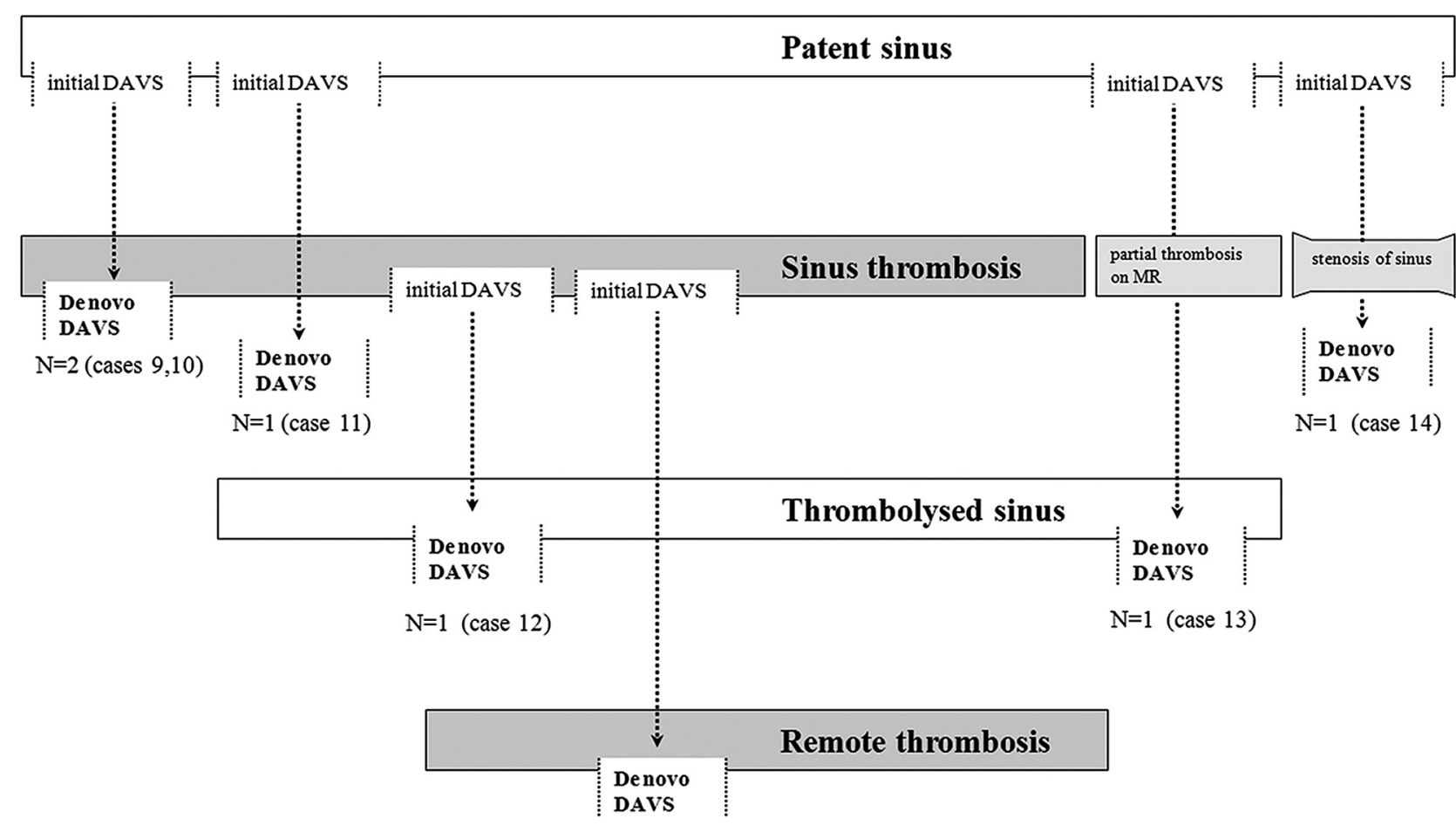

$\mathrm{N}=1$ (case 8)

Fig 1. Summary of the temporal sequence of de novo metachronous DAVS development in relation to sinus thrombosis.

Simultaneous sinus thrombosis and de novo lesion development were discovered in 2 patients (cases 9 and 10). The other 2 metachronous lesions without sinus occlusion on angiography showed partial thrombosis of the sinus on serial MR images (case 13) or stenosis of the sinus on serial angiograms before de novo DAVS development (case 14).

For all lesions, the priority of treatment was placed on the lesion with CVR. Complete occlusion of the shunt with CVR was achieved by transarterial and/or transvenous embolizations in 5 patients with synchronous DAVSs. Partial occlusion was achieved in 1 patient with CVR due to failure of the transvenous approach (case 1). One patient refused treatment (case 7 ). In the metachronous type, the initial lesions had been treated by transarterial embolization $(n=2)$, transvenous embolization $(n=1)$, and combined transarterial and transvenous embolizations $(n=4)$, resulting in complete occlusion in 4 patients. The de novo metachronous lesions were treated by transarterial embolization $(n=1)$, transvenous embolization $(n=1)$, transarterial and transvenous embolization $(n=$ 3 ), and Onyx (ev3, Irvine, California) embolization $(n=2)$, resulting in complete occlusion in 4 patients. Transvenous microcatheter manipulation had been performed in the sinus in which the de novo lesion developed in 4 of the 7 patients (cases $9,10,12$, and 14).

Overall, multiplicity of DAVSs was associated with aggressive initial symptoms such as neurologic deficit, seizure, and hemorrhage in $64.3 \%$ ( 9 of 14 patients). The synchronous type was associated with aggressive initial symptoms in $71.4 \%$ (5 of 7 patients). Hemorrhagic presentation was seen in 1 patient (case 5). In the metachronous type, initial hemorrhagic presentation was noted in 1 patient (case 10). Other initial symptoms were neurologic deficits $(n=2)$, seizure $(n=1)$, and benign symptoms $(n=3)$. The de novo lesions presented with hemorrhage $(n=1$, case 8$)$, neurologic deficit $(n=1$, case 12$)$, and worsening of the initial benign symptoms $(n=2$, cases 9 and 10). In case 8 , delayed rehemorrhage occurred after transarterial partial occlusion of the de novo lesion. The patient was later cured by burr-hole puncture and sinus occlusion. In case 12 , delayed hemorrhage was seen after detection of the de novo lesion before treatment. The de novo lesions were incidentally detected in the other 3 patients (cases 11, 13, and 14). Two of these incidental de novo lesions showed CVR (cases 11 and 14). The mean time interval between the initial diagnosis and de novo lesion detection was $31.3 \pm 29.8$ months (range, 12-92 months).

\section{Discussion}

The results of our series reveal the aggressive angiographic and clinical characteristics of multiple DAVSs and the prominent role of sinus thrombosis in the various pathomechanisms of DAVSs.

Thirteen of the 14 patients in our series showed CVR on the initial DSA. This high incidence of CVR associated with a multiplicity of DAVSs has been reported in other studies. ${ }^{7,15}$ It may be due to venous hypertension enhanced by the multiple DAVSs. ${ }^{14}$ There was no specific pattern of multiplicity in terms of the location and the interval of the de novo shunt development in our series. No specific sinus was immune from multiplicity, and there was no ipsilateral laterality as reported by Kubo et al. ${ }^{18}$ Our angiographic analysis showed that the de novo shunts had a tendency to occur in association with sinus thrombosis, frequently at the edges of the thrombosed sinuses. Prior history of transvenous embolization and the venous manipulation of microcatheters and coils with activation of an- 

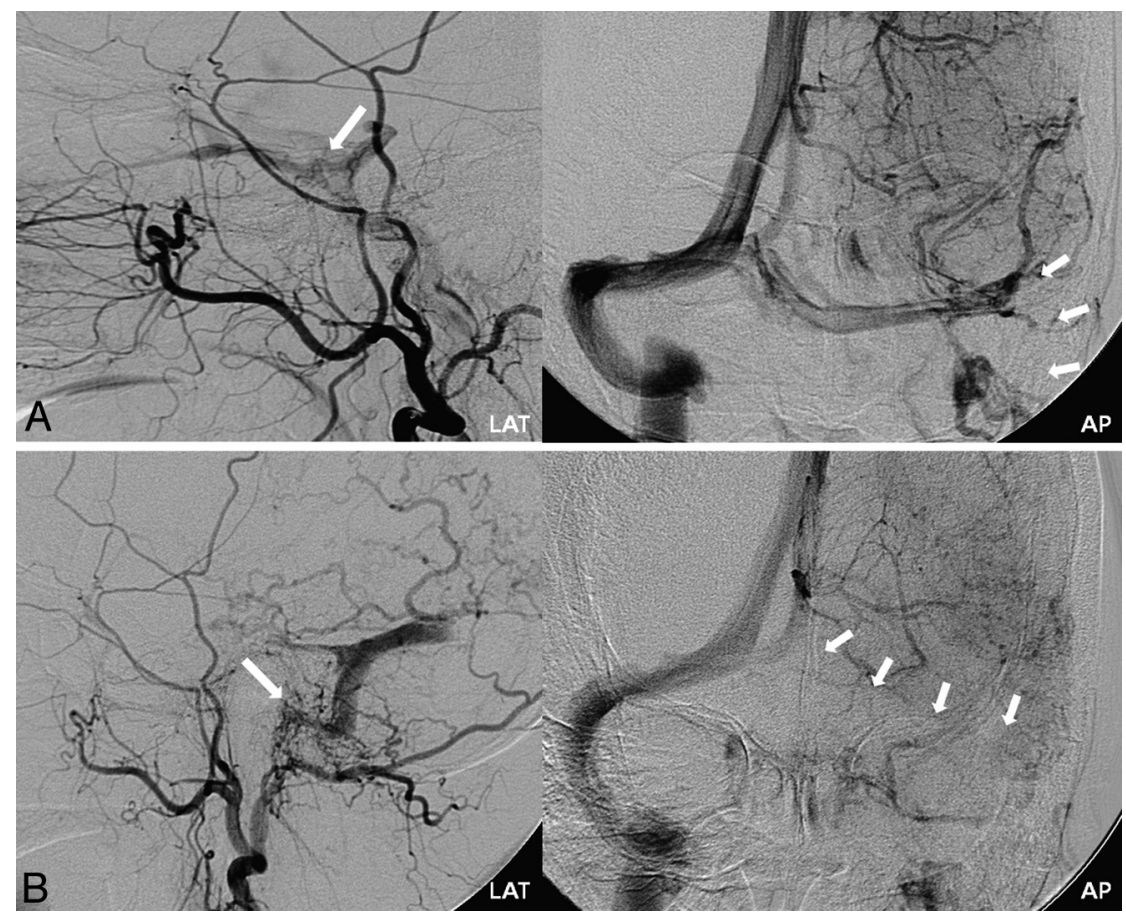

Fig 2. Case 8. A, Initial left external carotid artery (ECA) lateral and left ICA anteroposterior (AP) delayed phase angiograms. Cavernous DAVS (large arrow) is seen with occlusion of the left sigmoid sinus (small arrows). Transarterial embolization of the cavernous DAVS with polyvinyl alcohol particles was performed, resulting in complete occlusion. $B$, The patient presented with hemorrhage 17 months later. Follow-up left ECA lateral and left ICA AP delayed phase angiograms show de novo DAVS development at the previously occluded left sigmoid sinus (large arrow), with recanalization of this segment of the sinus. Upstream thrombosis of previously patent left proximal transverse sinus (small arrows) is seen, resulting in isolation of the de novo shunt.

giogenetic factors have been implicated in the development of de novo DAVSs through sinus thrombosis and venous obstruction in other series. ${ }^{19,20}$ In our series, 4 of the 7 metachronous lesions were associated with prior intrasinus manipulation.

The results of our study show the versatile feature of sinus thrombosis in the pathomechanism of multiple DAVSs. Venous sinus thrombosis adjacent to the shunt was seen in 5 of the synchronous cases, suggesting that either the sinus thrombosis induced the second shunt or the development of the second shunt induced the sinus thrombosis. The temporal sequential findings of the metachronous cases suggest the role of sinus thrombosis in the pathogenesis of this disease. Venous thrombosis may be associated with the breakdown of autoregulation of dural microvascularization and activation of the angiogenetic factor, resulting in DAVS development. ${ }^{22}$ Three patients showed development of de novo DAVSs adjacent to the occluded sinuses, which had been patent on the initial study. The presence of thrombus on the angiogram/MR images in these patients before shunt development also suggests the pathogenic potential of sinus thrombosis.

On the other hand, the development of a new shunt was also associated with remote sinus thrombosis formation (Fig 2). The presence of a shunt with reflux into the adjacent sinus may cause hemodynamic turbulence with the normal draining blood, leading to sinus thrombosis. ${ }^{14,22}$ Two patients showed new DAVSs with recanalization of a previously occluded sinus. Although sinus thrombus may be the cause of new shunt development, these cases show that the thrombus may also regress or recanalize after the development of the DAVS, which may be caused by the natural thrombolytic potency of circulating blood. Not all cases in our series showed visible sinus thrombus in relation to multiplicity. At least 2 cases showed sinus patency without any stenosis or occlusion. However, pathologic studies have shown that despite the angiographic evidence of sinus patency, thrombus can often be identified histologically in DAVSs. ${ }^{23}$

In addition to its pathogenic potential, sinus thrombosis is also known to be associated with spontaneous healing or pattern conversion of DAVSs. ${ }^{2}$ Depending on the underlying venous topography, the upstream or downstream development of sinus thrombus may alter the venous outflow pattern or may be associated with spontaneous cure.

Although the DAVSs in children and youth (cases 10 and 11) may be congenital in origin compared with the acquired lesions in adults, our experience shows that the pathomechanism of de novo DAVS development involving thrombosis of a previously patent dural sinus is similar in both children and adults.

The multiplicity of DAVSs was associated with a high ratio of aggressive initial symptoms, such as hemorrhage, neurologic deficit, or seizure in our series $(64.3 \%)$. The multiplicity of lesions by itself may not be directly associated with aggressive symptoms. However, these lesions were associated with a high incidence of CVR in our series (13/14 cases). This is in accordance with the previous reports claiming that multiple lesions are related to a more aggressive clinical presentation and entail a higher risk of hemorrhage. ${ }^{7}$ van Dijk et $\mathrm{al}^{15}$ reported, on the basis of their 19 patients with synchronous multiple DAVSs, that the percentage of hemorrhage at presentation was 3 times higher in the multiple DAVS group. The aggressive behavior at presentation is attributable to the high 
rate of CVR. ${ }^{24}$ Also of interest is the relatively higher association of the de novo lesions with hemorrhage compared with the initial lesions in our metachronous group. Two of the 7 de novo lesions were associated with hemorrhagic presentation ( $n=1$, case 8$)$ or hemorrhage before treatment ( $n=1$, case 12). The unstable dynamic changes of the venous flow pattern in these de novo lesions in association with CVR may have been a contributing factor for hemorrhage.

The high ratio of patients with synchronous and metachronous multiple DAVSs showing aggressive angiographic and clinical patterns underscores the importance of aggressive management for these patients. An important observation in the metachronous group in our series is that despite the high association of the de novo lesions with CVR, 3 of 7 lesions were not associated with any change in subjective symptoms/signs and were discovered on routine follow-up. Performing additional investigations in patients with DAVS who reveal a change in symptoms has been generally accepted. ${ }^{2}$ However, on the basis of our results, it may be prudent to perform periodic angiographic follow-up studies, especially in those cases with evidence of sinus thrombosis due to the risks of silent aggressive metachronous lesion development. Noninvasive studies such as time-resolved MRA imaging may be a safe and effective technique for follow-up. ${ }^{25}$

\section{Conclusions}

Multiplicity of DAVSs is associated with poor angiographic and clinical prognosis requiring an aggressive treatment and management strategy. Sinus thrombosis has a prominent role in both the cause and the consequence of DAVSs.

\section{References}

1. Berenstein A, Lasjaunias PL, Ter Brugge K. Dural Arteriovenous Shunts. Berlin, Germany: Springer-Verlag; 2004

2. Kim DJ, terBrugge K, Krings T, et al. Spontaneous angiographic conversion of intracranial dural arteriovenous shunt: long-term follow-up in nontreated patients. Stroke 2010;41:1489-94

3. Al-Shahi R, Bhattacharya JJ, Currie DG, et al. Prospective, population-based detection of intracranial vascular malformations in adults: the Scottish intracranial vascular malformation study (SIVMS). Stroke 2003;34:1163-69

4. Chaudhary MY, Sachdev VP, Cho SH, et al. Dural arteriovenous malformation of the major venous sinuses: an acquired lesion. AJNR Am J Neuroradiol 1982;3:13-19
5. van Dijk JM, TerBrugge KG, Van der Meer FJ, et al. Thrombophilic factors and the formation of dural arteriovenous fistulas. J Neurosurg 2007;107:56-59

6. Kusaka N, Sugiu K, Katsumata A, et al. The importance of venous hypertension in the formation of dural arteriovenous fistulas: a case report of multiple fistulas remote from sinus thrombosis. Neuroradiology 2001;43:980-84

7. Barnwell SL, Halbach VV, Dowd CF, et al. Multiple dural arteriovenous fistulas of the cranium and spine. AJNR Am J Neuroradiol 1991;12:441-45

8. Aminoff MJ. Vascular anomalies in the intracranial dura mater. Brain 1973;96:601-12

9. Barnwell SL, Halbach VV, Dowd CF, et al. Dural arteriovenous fistulas involving the inferior petrosal sinus: angiographic findings in six patients. AJNR Am J Neuroradiol 1990;11:511-16

10. Grady MS, Pobereskin L. Arteriovenous malformations of the dura mater. Surg Neurol 1987;28:135-40

11. Kataoka K, Taneda M. Angiographic disappearance of multiple dural arteriovenous malformations: case report. J Neurosurg 1984:60:1275-78

12. Kuwayama N, Takaku A, Nishijima M, et al. Multiple dural arteriovenous malformations: report of two cases. J Neurosurg 1989:71:932-34

13. Kubota Y, Ueda T, Kaku Y, et al. Development of a dural arteriovenous fistula around the jugular valve after transvenous embolization of cavernous dural arteriovenous fistula. Surg Neurol 1999;51:174-76

14. Ushikoshi S, Kikuchi Y, Houkin K, et al. Multiple dural arteriovenous fistulas. Neurol Med Chir (Tokyo) 1998;38:478-84

15. van Dijk JM, TerBrugge KG, Willinsky RA, et al. Multiplicity of dural arteriovenous fistulas. J Neurosurg 2002;96:76-78

16. Nakagawa H, Kubo S, Nakajima Y, et al. Shifting of dural arteriovenous malformation from the cavernous sinus to the sigmoid sinus to the transverse sinus after transvenous embolization: a case of left spontaneous carotid-cavernous sinus fistula. Surg Neurol 1992;37:30-38

17. Yamashita K, Taki W, Nakahara I, et al. Development of sigmoid dural arteriovenous fistulas after transvenous embolization of cavernous dural arteriovenous fistulas. AJNR Am J Neuroradiol 1993;14:1106-08

18. Kubo M, Kuwayama N, Hirashima Y, et al. Dural arteriovenous fistulae developing at different locations after resolution of previous fistulae: report of three cases and review of the literature. AJNR Am J Neuroradiol 2002;23: 787-89

19. Kiyosue H, Tanoue S, Okahara M, et al. Recurrence of dural arteriovenous fistula in another location after selective transvenous coil embolization: report of two cases. AJNR Am J Neuroradiol 2002;23:689-92

20. Gupta R, Horowitz M, Tayal A, et al. De novo development of a remote arteriovenous fistula following transarterial embolization of a carotid cavernous fistula: case report and review of the literature. AJNR Am J Neuroradiol 2005;26:2587-90

21. Borden JA, Wu JK, Shucart WA. A proposed classification for spinal and cranial dural arteriovenous fistulous malformations and implications for treatment. J Neurosurg 1995;82:166-79

22. Mironov A. Pathogenetical consideration of spontaneous dural arteriovenous fistulas (DAVFS). Acta Neurochir (Wien) 1994;131:45-58

23. Uranishi R, Nakase H, Sakaki T. Expression of angiogenic growth factors in dural arteriovenous fistula. J Neurosurg 1999;91:781-86

24. Awad IA, Little JR, Akarawi WP, et al. Intracranial dural arteriovenous malformations: factors predisposing to an aggressive neurological course. J Neurosurg 1990;72:839-50

25. Farb RI, Agid R, Willinsky RA, et al. Cranial dural arteriovenous fistula: Diagnosis and classification with time-resolved MR angiography at 3T. AJNR Am J Neuroradiol 2009;30:1546-51 